\title{
An Annotation Management System for Relational Databases
}

\author{
Deepavali Bhagwat Laura Chiticariu Wang-Chiew Tan* Gaurav Vijayvargiya \\ University of California, Santa Cruz \\ Email: \{dbhagwat, laura,wctan, gaurav\}@cs.ucsc.edu
}

\begin{abstract}
We present an annotation management system for relational databases. In this system, every piece of data in a relation is assumed to have zero or more annotations associated with it and annotations are propagated along, from the source to the output, as data is being transformed through a query. Such an annotation management system is important for understanding the provenance and quality of data, especially in applications that deal with integration of scientific and biological data.

We present an extension, pSQL, of a fragment of SQL that has three different types of annotation propagation schemes, each useful for different purposes. The default scheme propagates annotations according to where data is copied from. The default-all scheme propagates annotations according to where data is copied from among all equivalent formulations of a given query. The custom scheme allows a user to specify how annotations should propagate. We present a storage scheme for the annotations and describe algorithms for translating a pSQL query under each propagation scheme into one or more SQL queries that would correctly retrieve the relevant annotations according to the specified propagation scheme. For the default-all scheme, we also show how we generate finitely many queries that can simulate the annotation propagation behavior of the set of all equivalent queries, which is possibly infinite. The algorithms are implemented and the feasibility of the system is demonstrated by a set of experiments that we have conducted.
\end{abstract}

\section{Introduction}

For many scientifi c domains, new databases are often created to support the data analysis needs of domain-specifi c scientists. Some examples of such databases from biology include UniProt [2] and SWISS-PROT [3]. Data that is collected from other sources is often cleansed and reformatted

* Supported in part by an NSF CAREER Award IIS-0347065.

Permission to copy without fee all or part of this material is granted provided that the copies are not made or distributed for direct commercial advantage, the VLDB copyright notice and the title of the publication and its date appear, and notice is given that copying is by permission of the Very Large Data Base Endowment. To copy otherwise, or to republish, requires a fee and/or special permission from the Endowment.

Proceedings of the 30th VLDB Conference,

Toronto, Canada, 2004 before it is compiled into the new database. Very often, the newly created database will also contain new analysis or results that are derived by scientists. By associating old and new data together in the new database, an integrated perspective is provided to scientists and this is critical for further analysis and scientifi c discovery. With the proliferation of many such inter-dependent databases ${ }^{1}$, it is natural to ask what is the provenance of a piece of data (i.e., where that piece of data is copied or created from) in a database. Understanding the provenance of data is important towards understanding the quality of data which may help, for example, a scientist to decide on the amount of trust to place on a piece of information that she encounters in a database. We use the term annotations to mean information about data such as provenance, comments, or other types of metadata.

We describe an annotation management system for relational databases where annotations may be attached to a piece of data and are transparently carried along as data is being transformed. One immediate application is to use annotations to systematically trace the provenance and flow of data: if we attach to every piece of source data an annotation that describes its address (i.e., origins), then the annotations of a piece of data in the output of a transformation describe its provenance. Even if the data had undergone several transformation steps, we can easily determine the origins (or the flow of data for that matter) through the transformation steps by examining the annotations. Another use of annotations is to describe information about data that would otherwise have not been kept in a database. For example, an error report or remarks about a piece of data may be attached and propagated along to other databases, thus notifying other users of the error or additional information. The quality or security level of a piece of data can also be described in annotations. Since annotations are propagated along as a query is executed, the annotations on the result of a query can be aggregated to determine the quality or degree of sensitivity of the resulting output. This idea of using annotations to describe the security level of various data items or to specify fi negrained access control policies is not new and can be found in various forms in existing literature $[11,13,19]$.

We describe three propagation schemes for propagating

\footnotetext{
${ }^{1}$ See [10] for a catalog of biology databases.
} 
annotations that are motivated by different needs. They correspond to the default, default-all, and custom propagation schemes. The default scheme uses provenance as the basis for propagating annotations. If an output piece of data $d^{\prime}$ is copied from an input piece of data $d$, then the annotations associated with $d$ are propagated to $d^{\prime}$. A piece of output data $d^{\prime}$ is copied from an input piece of data $d$ if $d^{\prime}$ is created from $d$ according to the syntax and evaluation of the query. Although this natural defi nition corresponds intuitively to how people reason about provenance, the way annotations are propagated is dependent on the way a query is written. As shown in [24], two equivalent queries may propagate annotations differently. While this behavior may seem disturbing at first, in many applications including those described above, such an automatic provenance-based annotation propagation scheme which allows one to trace where data is copied from or copied to based on a given query is still very desirable. Indeed, similar ideas were proposed before in $[18,26]$. An alternative method of propagating annotations, called the default-all scheme, is to propagate annotations according to where data is copied from in all equivalent formulations of the given query since one may be interested in obtaining all relevant annotations of a piece of data in the output regardless of how a query may have been written. Unlike the default scheme, two equivalent queries will always propagate annotations in the same way under this scheme. In some cases, a user may only be interested in annotations provided by a certain trusted data source. Hence we also have a third propagation scheme, the custom propagation scheme, where the user is free to specify how annotations should be propagated.

Summary of results We have implemented all three propagation schemes in our annotation management system by extending a fragment of SQL. We call this extension $\mathrm{pSQL}$. A pSQL query is essentially an SQL query extended with a PROPAGATE clause that would propagate annotations according to one of the schemes described above as data is transformed. In our implementation, we assume that there is an "additional column" that stores the annotations for every attribute of every relation. A translation algorithm translates a given pSQL query into one or more SPJ queries against these underlying relations and these SPJ queries will retrieve the relevant annotations according to the specifi ed propagation scheme. In the default-all scheme, we are required to propagate annotations according to every possible equivalent reformulations of a given query. At fi rst sight, the default-all scheme seems impossible to implement as there are infi nitely many equivalent reformulations of a given query. We show, however, that it is always possible to fi nd a fi nite set of equivalent queries whose annotation propagation behavior is "representative" of all equivalent queries. Hence, by running every query in this fi nite set and taking the union of resulting tuples and annotations, we are able to obtain the annotated output of the given query under the default-all scheme. We have conducted experiments to evaluate the feasibility of such an annotation management system. Our experimental results indicate that the execution time of a query under any propagation scheme increases only slightly when the number of annotations in a database is doubled. Our results also show that for the queries we executed, the performance of a query under the default-all scheme can be at worst eight times slower than the performance of the same query under the default or no propagation scheme (i.e., SQL query). At best, it runs about twice as slow. For the default scheme, however, the execution times of $\mathrm{pSQL}$ queries are comparable to those of SQL queries. On the average, the pSQL queries with default scheme that we experimented with on a $100 \mathrm{MB}$ database took around $40 \%$ more time to execute than their corresponding SQL queries. For larger databases (500MB and 1GB), the pSQL queries with default scheme took only about $18 \%$ more time to execute than their corresponding SQL queries on the average.

Related Work The problem of computing data provenance is not new. Cui, Widom, and Wiener [9] fi rst approached the problem of tracing the provenance of data that is the result of a query applied on a relational database. The solution proposed in [9] was to fi rst generate a "reverse" query $Q^{r}$ when asked to compute the provenance of an output tuple $t$ in the result of a query $Q$ applied on a database $D$ (i.e., $Q(D)$ ). The result of applying $Q^{r}$ on $D$ consists of all combinations of source tuples in $D$ such that each combination of source tuples and $Q$ explain why $t$ is in the output of $Q(D)$. The type of provenance studied by [9] is called why-provenance according to Buneman, Khanna, and Tan [6]. Additionally, we may also be interested in knowing where the values of a tuple $t$ in the result of $Q(D)$ are copied from in $D$. The latter type of provenance is called where-provenance in [6] and it is this type of provenance that we use for determining where annotations are propagated from. In both works [6, 9], a "reverse" query is generated in order to answer provenance. While the reverse query approach works well in general, it requires a reverse query to be generated and evaluated every time the provenance of an output tuple is sought for. Hence if the provenance of a large number of output tuples is required, this may not be the optimal way to compute provenance.

The reverse query approach is what we call the lazy approach for computing provenance; a query is generated and executed to compute the provenance only when needed. In this paper, we propose to trade space for time and carry along the provenance of data as data is being transformed. Hence, in this approach, the provenance of data is eagerly computed and immediately available in the output. The idea of eagerly computing provenance by forwarding annotations along data transformations is also not new and has been proposed in various forms in existing literature $[4,18,26]$. In fact, our annotation propagation rules which propagate annotations based on where-provenance are similar to those proposed in [26]. In [26], however, only information about which source relations a value is copied from is propagated along. In contrast, our system is flexible in the amount of information that is carried along to the result (i.e., it could be the source relations, or the ex- 
act location within the source locations, or a comment on the data).

Numerous annotation systems have been built to support and manage annotations on text and HTML documents [14, 17, 21, 23, 25]. Recently, annotation systems for genomic sequences $[5,12,16]$ have also been built. Laliberte and Braverman [17] discussed how to use the HTTP protocol to design a scalable annotation system for HTML pages. Schickler, Mazer, and Brooks [23] discussed the use of a specialized proxy module that would merge annotations from an annotation store onto a Web page that is being retrieved before sending it to the client browser. Annotea $[14,25]$ is a W3C effort to support annotations on any Web document. Annotations are also stored on annotation servers and XPointer is used for pinpointing locations on a Web document. A specialized client browser that can understand, communicate, and merge annotations residing in the annotation servers with Web documents is used. Phelps and Wilensky [20, 21, 22] also discussed the use of annotations with certain desirable properties on multivalent documents [22] which support documents of different media types, such as images, postscript, or HTML. DAS or Biodas [5, 12] and the Human Genome Browser [16] are specialized annotation systems for genomic sequence data. In almost all of these systems, the design includes multiple distributed annotation servers for storing annotations and data is merged from various sources to display it graphically to an end user. The research of these systems has been focussed on the scalability of design, distributed support for annotations, or other added features.

We designed and implemented an annotation management system for relational databases where annotations can be made on relational data. This idea was fi rst proposed in [7, 24]. Unlike Web pages, the rigid structure of relations makes it easy to describe the exact position where an annotation is attached. Web pages, however, are often retrieved in part or as a whole. Hence, the issue of what annotations to propagate along when a web page is retrieved is straightforward. In contrast, an annotated relation in our system may undergo a complex transformation as a result of executing a query. We are thus concerned with how annotations should propagate when such complex transformations occur. To the best of our knowledge, this is the fi rst implementation of an annotation management system for relational databases that would allow a user to specify how annotations should propagate.

In Section 2, we describe pSQL and the three different propagation schemes. In Section 3, we describe the algorithm for generating a fi nite set of queries that can simulate the annotation propagation behavior of all equivalent queries of a given pSQL query. In Section 4, we describe the architecture of our system and a storage scheme for annotations as well as our translation algorithm that rewrites a pSQL query into an SQL query against the underlying storage scheme. In Section 5, we describe our experimental results and in Sections 6 and 7, we conclude with some possible future extensions to our system.

\section{2 pSQL}

In our subsequent discussions, we focus on a fragment of SQL that corresponds to conjunctive queries with union [1] (also known as the Select-Project-Join-Union fragment of SQL). We extend this fragment of SQL with a PROPAGATE clause to allow users to specify how annotations should propagate.

Definition 2.1 A pSQL query is a query of the form $Q_{1}$ UNION ... UNION $Q_{k}, k>0$, where each $Q_{i}, i \in[1, k]$, is a $p Q L$ query fragment of the form shown below:

$\begin{array}{ll}\text { SELECT DISTINCT } & \text { selectlist } \\ \text { FROM } & \text { fromlist } \\ \text { WHERE } & \text { wherelist } \\ \text { PROPAGATE } & \text { DEFAULT | DEFAULT-ALL | } \\ & r_{1} \cdot A_{1} \text { TO } B_{1}, \ldots, r_{n} . A_{n} \text { TO } B_{n}\end{array}$

The fromlist of a pSQL query fragment is of the form " $R_{1} r_{1}, \ldots, R_{k} r_{k}$ " where $r_{i}$ is a tuple variable of the corresponding relation $R_{i}$. The selectlist of a pSQL query fragment is of the form " $r_{1} . C_{1}$ AS $D_{1}, \ldots, r_{m} . C_{m}$ AS $D_{m}$ " where $r_{i}$ is a tuple variable defi ned in fromlist, $C_{i}$ is an attribute of the relation that corresponds to $r_{i}$, and $D_{i}$ is an attribute name of the output relation. The WHERE clause is optional and the wherelist is a conjunction of one or more equalities between attributes of relations or between attributes of relations and constants. The PROPAGATE clause can be defi ned with DEFAULT, DEFAULT-ALL, or a list of clauses of the form " $r$. $A$ TO $B$ " defi nitions where $r$. $A$ denotes an attribute $A$ of the tuple that is bound to $r$ and $B$ is an attribute among the $D_{j} \mathrm{~s}$.

The $S Q L$ query that corresponds to a $p S Q L$ query $Q$ is the SQL query that results when all PROPAGATE clauses in $Q$ have been removed. The meaning of a pSQL query is similar to that of its corresponding SQL query except that annotations are also propagated to each emitted tuple according to the specifi cation given in the PROPAGATE clauses.

Example 2.1 Consider three databases SWISS-PROT (a protein database), PIR (another protein database), and Genbank (a gene database). Each of these databases is modeled as a relation. The schemas and an instance of each relation are shown in Figure 1 (ignore the rest of the relations in the fi gure). An annotation, shown in braces, is placed on every column of every tuple. Each annotation can be interpreted as the address of the value in the corresponding column of the tuple. An example of a pSQL query with the default propagation scheme is shown below.

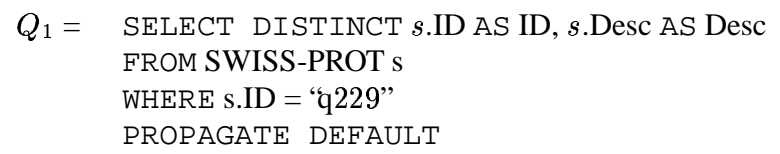

Intuitively, the default scheme specifi ed in $Q_{1}$ propagates annotations of data according to where data is copied from. The result of $Q_{1}$ executed against the relation SWISS-PROT is shown in Figure 1. The annotation $a_{3}$ is attached to the value q229 in the output since q229 is copied from the ID attribute of the second tuple in SWISSPROT. Likewise, $a_{4}$ in the output is propagated from the 


\section{SWISS-PROT}

\begin{tabular}{|l|l|}
\hline ID & Desc \\
\hline \hline z131 $\left\{a_{1}\right\}$ & AB $\left\{a_{2}\right\}$ \\
\hline q229 $\left\{a_{3}\right\}$ & CC $\left\{a_{4}\right\}$ \\
\hline q939 $\left\{a_{5}\right\}$ & ED $\left\{a_{6}\right\}$ \\
\hline
\end{tabular}

Mapping_Table

\begin{tabular}{|l|l|l|l|}
\hline entryid & swissprot & pir & genbank \\
\hline \hline $1\left\{a_{15}\right\}$ & z131 $\left\{a_{16}\right\}$ & p332 $\left\{a_{17}\right\}$ & g231 $\left\{a_{18}\right\}$ \\
\hline $2\left\{a_{19}\right\}$ & q229 $\left\{a_{20}\right\}$ & p916 $\left\{a_{21}\right\}$ & g756 $\left\{a_{22}\right\}$ \\
\hline $3\left\{a_{23}\right\}$ & q939 $\left\{a_{24}\right\}$ & p677 $\left\{a_{25}\right\}$ & g635 $\left\{a_{26}\right\}$ \\
\hline
\end{tabular}

PIR
\begin{tabular}{|l|l|}
\hline ID & Name \\
\hline \hline p332 $\left\{a_{7}\right\}$ & $\mathrm{AB}\left\{a_{8}\right\}$ \\
\hline p916 $\left\{a_{9}\right\}$ & $\mathrm{AB}\left\{a_{10}\right\}$ \\
\hline
\end{tabular}

Figure 1: Three protein databases, a mapping table, and three annotated outputs.

annotation of the Desc attribute of the second tuple in SWISS-PROT.

While the default scheme is a natural scheme for propagating annotations, this scheme is not "robust" in that two equivalent queries that return the same output may not propagate the same annotations to the output.

Example 2.2 Consider two equivalent SQL queries $Q^{\prime}$ and $Q^{\prime \prime}$ (two queries are equivalent if they produce the same result on every database).

$$
\begin{aligned}
Q^{\prime}=\quad & \text { SELECT DISTINCT } p . \text { ID AS ID, } p . \text { Name AS Name } \\
& \text { FROM PIR } p \text {, Mapping_Table } m \\
& \text { WHERE } p . \text { ID }=m \text {.pir } \\
Q^{\prime \prime}=\quad & \text { SELECT DISTINCT } m \text {.pir AS ID, } p \text {.Name AS Name } \\
& \text { FROM PIR } p, \text { Mapping_Table } m \\
& \text { WHERE } p . \text { ID }=m \text {.pir }
\end{aligned}
$$

The results of running $Q^{\prime}$ and $Q^{\prime \prime}$ under the default propagation scheme are shown below.

Result of $Q^{\prime}$ :

\begin{tabular}{|l|l|}
\hline ID & Name \\
\hline \hline $\mathrm{p} 332\left\{a_{7}\right\}$ & $\mathrm{AB}\left\{a_{8}\right\}$ \\
\hline $\mathrm{p} 916\left\{a_{9}\right\}$ & $\mathrm{AB}\left\{a_{10}\right\}$ \\
\hline
\end{tabular} \begin{tabular}{|l|l|}
\hline ID & Name \\
\hline \hline $\mathrm{p} 332\left\{a_{17}\right\}$ & $\mathrm{AB}\left\{a_{8}\right\}$ \\
\hline $\mathrm{p} 916\left\{a_{21}\right\}$ & $\mathrm{AB}\left\{a_{10}\right\}$ \\
\hline
\end{tabular}

For $Q^{\prime}$, the annotations for the ID column are from the PIR table while for $Q^{\prime \prime}$, the annotations for the ID column are from the Mapping_Table.

While it is likely that a user will realise that $Q^{\prime}$ will generate a different annotated outcome from $Q^{\prime \prime}$ in general, the situation is not so straightforward for more complex queries. The above example motivates the need for a propagation scheme that is invariant under equivalent queries. One should be able to retrieve all relevant annotations about a piece of output data regardless of how the query is written, if desired. The default-all propagation scheme propagates annotations according to where data is copied from among all equivalent formulations of the given query. Hence the annotated outcome is the same for equivalent queries under this scheme. In case a user prefers to retrieve annotations from one source over another, the user is also free to specify how annotations should propagate in the custom scheme.

Example 2.3 The queries $Q_{2}$ and $Q_{3}$ are examples of pSQL queries with the default-all and custom propagation schemes respectively.

Genbank
\begin{tabular}{|l|l|}
\hline ID & Desc \\
\hline \hline g231 $\left\{a_{11}\right\}$ & AB $\left\{a_{12}\right\}$ \\
\hline g756 $\left\{a_{13}\right\}$ & CC $\left\{a_{14}\right\}$ \\
\hline
\end{tabular}

Result of $Q_{1}$ :

\begin{tabular}{|l|l|}
\hline ID & Desc \\
\hline \hline q229 $\left\{a_{3}\right\}$ & CC $\left\{a_{4}\right\}$ \\
\hline
\end{tabular}

Result of $Q_{2}$ :

\begin{tabular}{|l|l|}
\hline ID & Name \\
\hline \hline p332 $\left\{a_{7}\right\}$ & $\mathrm{AB}\left\{a_{8}, a_{10}\right\}$ \\
\hline p916 $\left\{a_{9}\right\}$ & $\mathrm{AB}\left\{a_{8}, a_{10}\right\}$ \\
\hline
\end{tabular}$\quad$\begin{tabular}{|l|l|}
\hline ID & Desc \\
\hline \hline g231 $\left\{a_{11}, a_{12}\right\}$ & AB \\
\hline
\end{tabular}

Result of $Q_{3}$ :

$$
\begin{aligned}
Q_{2}= & \text { SELECT DISTINCT } p . \text { ID AS ID, } p \text {.Name AS Name } \\
& \text { FROM PIR } p \\
& \text { PROPAGATE DEFAULT-ALL } \\
Q_{3}= & \text { SELECT DISTINCT } g . \text { ID AS ID, } g \text {.Desc AS Desc } \\
& \text { FROM Genbank } g \\
& \text { PROPAGATE } g . \text { ID TO ID, } g \text {.Desc TO ID }
\end{aligned}
$$

The results of $Q_{2}$ and $Q_{3}$ are shown in Figure 1. The query $Q_{2}$ retrieves all tuples from the PIR table under the default-all propagation scheme. The annotations we get in the result are the combined annotations of results from all equivalent queries. In the custom scheme of $Q_{3}$, annotations are propagated according to the given user specifi cation (i.e., $g$.ID TO ID, $g$.Desc TO ID). A clause " $g$.ID TO ID" states that the annotations associated with the value of the ID attribute of the tuple that is currently bound to $g$ should propagate to the ID attribute of the output tuple. Similarly, the annotations associated to the value of the Desc attribute of the tuple that is currently bound to $g$ should propagate to the ID attribute of the output tuple.

Some Terminology A cell (or location) is a triple $(r, t$, $i$ ) which denotes the $i$ th column of the tuple $t$ in relation $r$. We sometimes use the attribute name at position $i$ instead of the position $i$. We also write a cell simply as a pair $(t, i)$ in the context where the relation $r$ is clear. Each cell contains a value of some type. We use $v(c)$ to denote the value at cell $c(v(c)$ is called a piece of data). Let $\mathcal{L}$ denote the set of all strings. Each cell $c$ in a database is associated with a set of annotations $\left\{a_{1}, \ldots, a_{k}\right\}$ where each $a_{i}, i \in[1, k]$, is an element in $\mathcal{L}$. We also say each $a_{i}, i \in[1, k]$, is an annotation attached to $c$. We use the notation $\mathcal{A}(c)$ to denote the set of all annotations attached to cell $c$.

Containment vs. Annotation-Containment. Two pSQL queries $Q$ and $Q^{\prime}$ are equivalent, denoted as $Q=Q^{\prime}$, if for every database $D, Q(D)=Q^{\prime}(D)$. The query $Q$ is contained in $Q^{\prime}$, denoted as $Q \subseteq Q^{\prime}$, for every database $D, Q(D) \subseteq Q^{\prime}(D)$. Two pSQL queries $Q$ and $Q^{\prime}$ are annotation-equivalent, denoted as $Q={ }_{a} Q^{\prime}$, if $Q$ and $Q^{\prime}$ produce the same annotated output on all databases. More precisely, this means that for every database $D, Q(D)$ is equal to $Q^{\prime}(D)$ and the set of annotations $\mathcal{A}((Q(D), t, i))$ is identical to $\mathcal{A}\left(\left(Q^{\prime}(D), t, i\right)\right)$ for every output location $(t, i)$ in $Q(D)$. A pSQL query $Q$ is annotation-contained in $Q^{\prime}$, denoted as $Q \subseteq \subseteq_{a} Q^{\prime}$, if for every database $D$, $Q(D) \subseteq Q^{\prime}(D)$ and for every output location $(t, i)$ in $Q(D), \overline{\mathcal{A}}((Q(D), t, i)) \subseteq \mathcal{A}\left(\left(Q^{\prime}(D), t, i\right)\right)$. 
Example 2.4 Figure 1 shows several examples of annotated relations. The value z131 in SWISS-PROT is the value at cell (SWISS-PROT, (z131, AB), ID) which denotes the ID column of tuple (z131, AB) in the SWISSPROT relation. Note that the attribute names in the tuple (z131, AB) have been omitted. The annotation $\left\{a_{1}\right\}$ is the set of annotations associated with this cell. Hence, $\mathcal{A}((\mathrm{SWISS}-\mathrm{PROT},(\mathrm{z} 131, \mathrm{AB}), \mathrm{ID}))$ is $\left\{a_{1}\right\}$. In the result of $Q_{2}, \mathcal{A}(((\mathrm{p} 332, \mathrm{AB}), \mathrm{Name}))$ is $\left\{a_{8}, a_{10}\right\}$.

\subsection{The Custom Propagation Scheme}

We allow the user the flexibility to specify custom propagation schemes using a PROPAGATE clause of the form " $r_{1} . A_{1}$ TO $B_{1}, \ldots, r_{n} . A_{n}$ TO $B_{n}$ ". The semantics of a pSQL query fragment $Q$ with custom propagation scheme is as follows. For every binding $\mu$ of tuple variables to tuples in the respective relations according to the fromlist of $Q$ such that the conditions in the wherelist are satisfi ed, emit an output tuple $t$ according to the selectlist. For every clause " $r_{i} . A_{i}$ TO $B_{i}$ " specifi ed in the PROPAGATE clause, we add the set of annotations at the location $\left(r_{i}, A_{i}\right)$ to the set of annotations (initially empty) at the output location $\left(t, B_{i}\right)$. Finally, duplicate output tuples are merged. Suppose $t_{1}, \ldots, t_{k}$ are the emitted tuples and $s_{1}, \ldots, s_{m}$ are the tuples that result when duplicate output tuples have been merged. Then, for every output location $(s, B), \mathcal{A}((s, B))$ $=\bigcup_{t_{j}=s, j \in[1, k]} \mathcal{A}\left(\left(t_{j}, B\right)\right)$. The query $Q_{3}$ of Example 2.3 is an example of a pSQL query fragment with a custom propagation scheme; every tuple in Genbank is emitted in such a way that the set of annotations that is associated with the ID column of an output tuple is the union of annotations associated with the ID column and Desc column of the corresponding tuple in Genbank.

\subsection{The Default Propagation Scheme}

If PROPAGATE DEFAULT is used in a pSQL query fragment, the set of annotations of a piece of output data consists of all the annotations associated with where that piece of data is copied from in the source.

The semantics of a $\mathrm{pSQL}$ query fragment $Q$ with the default propagation scheme is as follows. For every binding of tuple variables to tuples in the respective relations according to the fromlist of $Q$ such that the conditions in the wherelist are satisfi ed, emit an output tuple $t$ according to the selectlist as well as the corresponding sets of annotations for every cell in $t$. Since every value of an output cell $c^{\prime}$ in $t$ is generated from some value of an input cell $c$ according to the current bindings, the set of annotations attached to $c$ is also attached to $c^{\prime}$. Finally, duplicate output tuples are merged together. Suppose $t_{1}, \ldots, t_{k}$ are the emitted tuples and $s_{1}, \ldots, s_{m}$ are the tuples that result when duplicate output tuples have been merged. Then, for every output location $(s, B), \mathcal{A}((s, B))$ $=\bigcup_{t_{j}=s, j \in[1, k]} \mathcal{A}\left(\left(t_{j}, B\right)\right)$.

Example 2.5 Suppose we have the following pSQL query where each fragment uses the default propagation scheme.

\begin{tabular}{lll|} 
SELECT & Desc AS Desc & \\
FROM & SWISS-PROT & Result: \\
PROPAGATE & DEFAULT & Desc \\
UNION & & $\mathrm{AB}\left\{a_{2}, a_{12}\right\}$ \\
SELECT & Desc AS Desc & $\mathrm{CC}\left\{a_{4}, a_{14}\right\}$ \\
FROM & Genbank & $\operatorname{ED}\left\{a_{6}\right\}$ \\
PROPAGATE & DEFAULT &
\end{tabular}

DEFAULT

The fi rst subquery emits an output tuple "AB" with annotations $\left\{a_{2}\right\}$ and the second subquery emits the same output tuple "AB" but with annotations $\left\{a_{12}\right\}$. The merged result of these two tuples is a single output tuple "AB" with annotations $\left\{a_{2}, a_{12}\right\}$. This explains the fi rst output tuple in the result. A similar reasoning applies to the rest of the output tuples.

It is easy to see that a pSQL query fragment with default propagation scheme can be translated into a pSQL query fragment with custom propagation scheme. For example, the query $Q_{1}$ of Example 2.1 can be rewritten into a $\mathrm{pSQL}$ query with custom scheme where the propagate clause is replaced by "PROPAGATE $s$.ID TO ID, $s$.Desc TO Desc" since the ID value and Desc value of an output tuple are copied from $s$.ID and $s$.Desc, respectively.

\subsection{The Default-All Propagation Scheme}

A pSQL query with the default propagation scheme is, essentially, an SQL query with annotations propagated based on where a value is retrieved according to the syntax of the query. We have already seen an example of two pSQL queries under the default propagation scheme (Example 2.2) which are equivalent but not annotationequivalent.

This motivates us to defi ne a third propagation scheme, called the default-all scheme, where the annotation propagation behavior of a pSQL query is invariant to the syntax of the query. A pSQL query $Q$ with default-all propagation scheme propagates annotations according to the default propagation behavior of all equivalent formulations of $Q$. The resulting tuples that are generated by all equivalent queries of $Q$ according to the default scheme are then merged together. Despite the fact that there are infi nitely many equivalent formulations of $Q$, we describe a method that would compute the desired result by examining only a finite number of $\mathrm{pSQL}$ queries. We call such a fi nite set of queries a query-basis of $Q$.

Definition 2.2 Let $Q$ denote a pSQL query with defaultall propagation scheme. Let $S(Q)$ denote the SQL query that corresponds to $Q$ and let $\mathcal{E}(S(Q))$ denote the set of all pSQL queries $Q^{\prime}$ under the default propagation scheme such that $S\left(Q^{\prime}\right)$ is equivalent to $S(Q)$. A query basis of $Q$, denoted as $\mathcal{B}(Q)$, is a fi nite set of pSQL queries with default propagation scheme such that $\bigcup_{q \in \mathcal{B}(Q)} q={ }_{a}$ $\bigcup_{q \in \mathcal{E}(S(Q))} q$.

We describe next an algorithm that fi nds a query basis for a $\mathrm{PSQL}$ query with default-all propagation scheme. The size of the query basis that the algorithm returns is always polynomial in the size of $Q$. 


\section{Generating a Query Basis}

The algorithm for computing a query basis for a $\mathrm{pSQL}$ query with default-all propagation scheme proceeds by fi rst generating a representative query of $Q$, called $Q_{0}$. Intuitively, a representative query of $Q$ is a query that is equivalent to $Q$ and for every attribute $A$ that is equal or transitively equal to an attribute $B$ in the selectlist of $Q$, the annotations of $A$ are propagated to $B$. From $Q_{0}$, a fi nite number of auxiliary queries are also generated and these queries, together with $Q_{0}$, form a query basis of $Q$. Each auxiliary query is equivalent to $Q$ but may propagate additional annotations to the output that are not propagated by $Q_{0}$. Intuitively, only a fi nite number of auxiliary queries are needed because only one auxiliary query needs to be generated for each attribute of a relation that "contributes annotations" to the output. In the rest of the discussion, we restrict our language to be $\mathrm{pSQL}$ query fragments. We present an algorithm for generating a query basis of a $\mathrm{pSQL}$ query fragment with default-all propagation scheme. The algorithm can be extended to handle PSQL queries in general and the details are omitted.

Algorithm Generate-Query-Basis

Input: A pSQL query fragment $Q$ with default-all propagation scheme.

Output: A query basis of $Q, \mathcal{B}(Q)$.

Let $Q$ be a pSQL query fragment of the form shown in Definition 2.1 with PROPAGATE DEFAULT-ALL clause.

1. Generate $Q_{0}$, the representative query of $Q$.

Generate a query $Q_{0}$ that is identical to $Q$ except that the propagation scheme of $Q$ is replaced with the following propagation scheme:

For every attribute " $r$. $A$ AS $C$ " in the selectlist, add " $r . A$ TO $C$ " in the PROPAGATE clause.

For every attribute " $r . A$ AS $C$ " in the selectlist and every attribute $s . B$ that is equal to $r$. $A$ or transitively equal to $r . A$ according to the wherelist: add "s.B TO $C$ " in the PROPAGATE clause.

(The effect is that all attributes that are equal to an attribute $C$ in the selectlist have their annotations propagated to $C$.)

2. Generate auxiliary queries of $Q_{0}$.

Initialize $\mathcal{B}(Q)$ to the empty set. Add $Q_{0}$ to $\mathcal{B}(Q)$. For every attribute "r. A AS $C$ " in the selectlist of $Q_{0}$ and every "s.B TO $C$ " in the PROPAGATE clause of $Q_{0}$, do the following:

Create a query $Q^{\prime}$ that is identical to $Q_{0}$. Suppose $s$ is a tuple variable of relation $S$ according to the fromlist of $Q_{0}$. Add " $S s^{\prime}$ " to the fromlist of $Q^{\prime}$ where $s^{\prime}$ is a tuple variable that does not occur in $Q^{\prime}$. Add " $s$ '. $B=s . B$ " to the wherelist of $Q$ ' and " $s$ '. $B$ TO $C$ " to the PROPAGATE clause of $Q^{\prime}$. Add $Q^{\prime}$ to $\mathcal{B}(Q)$.

(The auxiliary query $Q^{\prime}$ is equivalent to $Q$ but may carry additional annotations to the output.)

3. Return $\mathcal{B}(Q)$.

Example 3.1 Consider the three databases, SWISS-PROT, PIR, and Genbank along with a Mapping_table that contains the correspondences between identifi ers of genes and proteins in the three databases in Figure 1. Such mapping tables commonly occur in integrating many sources with overlapping information [15]. Suppose we have the following query $Q$ that integrates information from SWISS-PROT and PIR.

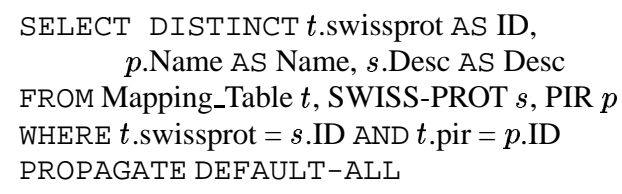

After Step 1 of the above algorithm, we obtain the following representative query $Q_{0}$ :

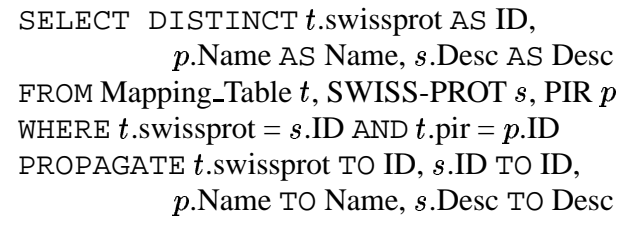

Note that the annotations of $t$.swissprot and $s$.ID will propagate to the output ID column according to $Q_{0}$. The second step of the algorithm generates four auxiliary queries. The fi rst query is shown below and the rest are shown in Figure 2.

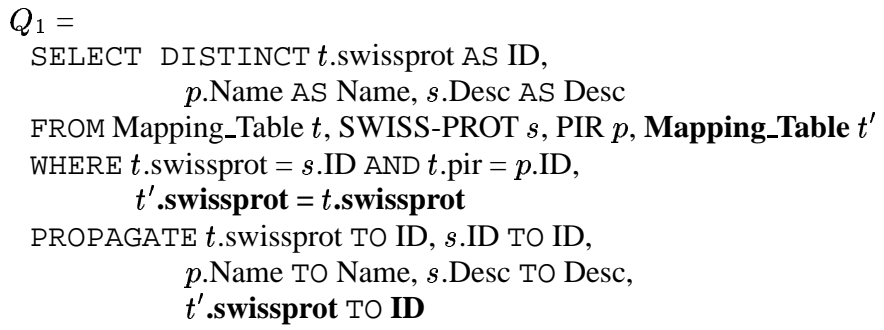

The query $Q_{1}$ is different from $Q_{0}$ only in the additional highlighted terms shown in $Q_{1}$. There is an extra relation, condition, and propagation in the FROM, WHERE, and PROPAGATE clauses respectively. It is easy to verify that the SQL queries of $Q_{0}$ and $Q_{1}$ are equivalent. There is a homomorphism $h$ from the tuple variables of $Q_{1}$ to those of $Q_{0}$ such that $h$ maps the fromlist of $Q_{1}$ to a subset of the fromlist of $Q_{0}$ and the conditions in the wherelist of $Q_{0}$ imply the conditions in the wherelist of $Q_{1}$ under $h$. Furthermore, $h$ maps the selectlist of $Q_{1}$ to the selectlist of $Q_{0}$. There is also a homomorphism in the reverse direction. Similarly, $Q_{2}, Q_{3}$, and $Q_{4}$ of Figure 2 are each equivalent to $Q_{0}$.

Intuitively, the representative query $Q_{0}$ propagates annotations according to where data is copied from and also where data could have been equivalently copied from. The reason why $Q_{0}$ is generated becomes clearer if we represent $Q$ using conjunctive query-like notation

$$
A(\mathbf{x}):-S_{1}\left(\mathbf{y}_{\mathbf{1}}\right), \ldots, S_{n}\left(\mathbf{y}_{\mathbf{n}}\right), \text { equalities. }
$$

where $\mathbf{x}, \mathbf{y}_{\mathbf{i}}, i \in[1, n]$, denote vectors of variables and every variable in $\mathbf{x}$ occurs in $\mathbf{y}_{\mathbf{i}}$ for some $i \in[1, n]$ and equalities is a list of zero of more $y=y^{\prime}$ clauses where $y$ is a variable that occurs amongst $\mathbf{y}_{\mathbf{i}}$ s and $y^{\prime}$ is a constant. The variables in $\mathbf{x}$ are called distinguished variables. Each subgoal corresponds to a relation in the fromlist of $Q$. The 


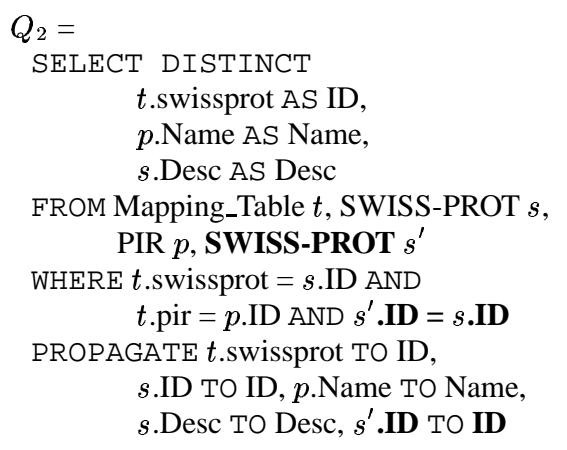

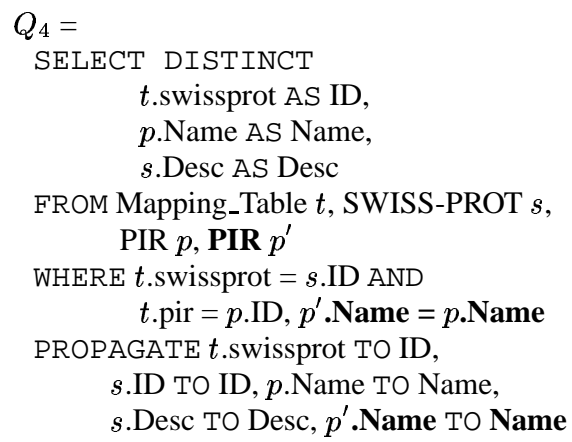

Figure 2: Some of the auxiliary queries generated by Step 2 of Generate-Query-Basis on Example 3.1.

equalities between attributes in the wherelist of $Q$ are represented by using the same variable in the respective positions of relations in the conjunctive query-like representation of $Q$. An equality between an attribute and constant is written out as equalities. The head of the query $A(\mathbf{x})$ represents the selectlist of $Q$. We use $C(Q)$ to denote the conjunctive query-like representation of the SQL query that corresponds to $Q$. For example, $C(Q)$ of Example 3.1 can be written as

$\mathrm{A}_{0}(x, y, z)$ :- Mapping_Table $(w, x, u, v), \operatorname{SWISS-PROT}(x, z)$, $\operatorname{PIR}(u, y)$.

Similar to the semantics of pSQL queries with the default propagation scheme, annotations are propagated according to where data is copied from for such queries [24] by tracing the occurrence of distinguished variables in the query. For example, by tracing the occurrence of the variable $x$ in the query $A_{0}$, we can conclude that the annotations in the first column of an output tuple $t$ is obtained from the annotations of the second column of a tuple in Mapping_Table and the fi rst column of a tuple in SWISSPROT that created $t$. A similar argument applies to the variables $y$ and $z$ in $A_{0}$. Hence, the representative query $Q_{0}$ of Example 3.1 is annotation-equivalent to $A_{0}$.

Proposition 3.1 The representative query $Q_{0}$ that is generated by Generate-Query-Basis $(Q)$ is annotationequivalent to $C\left(Q_{0}\right)$.

In Step 2 the algorithm generates one query for every position in the body where a distinguished variable occurs in $A_{0}$. For example, the following four auxiliary queries, in conjunctive query notation, are generated based on $A_{0}$. They are annotation-equivalent to the pSQL query fragments $Q_{1}, \ldots, Q_{4}$ shown in Example 3.1 and Figure 2, respectively.

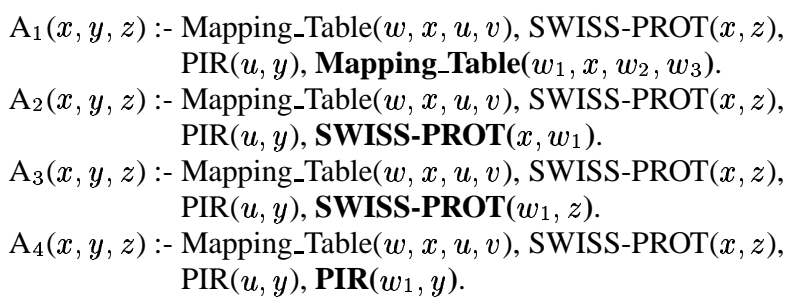

Proposition 3.2 For every query $Q^{\prime}$ in the result of Generate-Query-Basis $(Q)$ (denoted as $\mathcal{B}(Q)$ ), $C\left(Q^{\prime}\right)$ is annotation-contained in $\bigcup_{q \in \mathcal{B}(Q)} q$.
Each auxiliary query carries annotations to the output that may have been missed by the representative query of $Q$. We shall show next that the set of $\mathrm{pSQL}$ query fragments in $\mathcal{B}(Q)$ generated by the algorithm is a query basis for $Q$. We fi rst prove the following lemma.

Lemma 3.1 Let $\mathcal{B}(Q)$ denote the result of GenerateQuery-Basis $(Q)$ where $Q$ is a $p S Q L$ query fragment and let $Q^{\prime}$ denote a $p S Q L$ query fragment under the default propagation scheme. If $Q^{\prime}$ is equivalent to $Q$, then $Q^{\prime}$ is annotation-contained in $\bigcup_{q \in \mathcal{B}(Q)} q$.

Proof. We know from Proposition 3.1 that the representative query $Q_{0}$ that is generated at Step 1 of the algorithm is annotation-equivalent to the conjunctive query representation of the SQL query that corresponds to $Q, C(Q)$. We can also easily verify that $Q^{\prime} \subseteq \subseteq_{a} C\left(Q^{\prime}\right)$. Since $C(Q)$ and $C\left(Q^{\prime}\right)$ are equivalent queries, the minimal queries of $C(Q)$ and $C\left(Q^{\prime}\right)$ are identical up to variable renaming. For convenience, we shall assume that the minimal queries are identical in the form shown below. We also assume that there are no equalities between variables and constants, for convenience.

$C(Q): H(\mathbf{x})$ :- minpart, rest1.

$C\left(Q^{\prime}\right): H(\mathbf{x}):-$ minpart, rest 2 .

The subgoals denoted by minpart are the subgoals in the minimal query of $C(Q)$ or $C\left(Q^{\prime}\right)$ and rest 1 and rest2 denote the rest of the subgoals in $C(Q)$ and $C\left(Q^{\prime}\right)$, respectively. Our proof makes use of an earlier result in [24] extended for unions of conjunctive queries. Given a conjunctive query $Q$, we use the notation $Q[0]$ to denote the head of $Q$, the notation $Q[i], i>0$, to denote the $i$ th subgoal of $Q$, and $\operatorname{var}(Q[i])$ to denote the list of variables of the $i$ th subgoal of $Q$.

Fact 1 ([24]) Given two unions of conjunctive queries $Q=\bigcup_{i=1}^{m} Q_{i}$ and $Q^{\prime}=\bigcup_{j=1}^{n} Q_{j}^{\prime}, Q \subseteq_{a} Q^{\prime}$ if and only if for every $Q_{r}, r \in[1, m]$, and every variable $x$ that occurs at both the $i$ th position of $\operatorname{var}\left(Q_{r}[0]\right)$ and the $j$ th position of $\operatorname{var}\left(Q_{r}[p]\right)$ for some $p$, there exists a homomorphism $h$ from $Q_{s}^{\prime}$ (for some $s \in[1, n]$ ) to $Q_{r}$ such that

1. $h$ maps the body of $Q_{s}^{\prime}$ into the body of $Q_{r}$ and the head of $Q_{s}^{\prime}$ to the head of $Q_{r}$, and

2. the variable that occurs at the $j$ th position of the $q$ th subgoal of $Q_{s}^{\prime}$ (i.e., $\operatorname{var}\left(Q_{s}^{\prime}[q]\right)[j]$ ) is identical to the 
variable at the $i$ th position of the head of $Q_{s}^{\prime}$ (i.e., $\left.\operatorname{var}\left(Q_{s}^{\prime}[0]\right)[i]\right)$, where $Q_{s}^{\prime}[q]$ is a pre-image of $Q_{r}[p]$ under $h$. That is, for some subgoal $q, \operatorname{var}\left(Q_{s}^{\prime}[q]\right)[j]=$ $\operatorname{var}\left(Q_{s}^{\prime}[0]\right)[i]$ and $h\left(Q_{s}^{\prime}[q]\right)=Q_{r}[p]$.

We shall show that for every distinguished variable $x$ at the $i$ th position in the head of $C\left(Q^{\prime}\right)$ and its occurrence at the $j$ th position of the $p$ th subgoal $S(\mathbf{u})$ (i.e., the $j$ th variable of $\mathbf{u}$ is $x$ ) in the body of $C\left(Q^{\prime}\right)$, there is a generated query $Q_{g}$ in $\mathcal{B}(Q)$ and a homomorphism $h: C\left(Q_{g}\right) \rightarrow$ $C\left(Q^{\prime}\right)$ that satisfi es the conditions (1) and (2) stated in the fact. Then by the above fact, we have $C\left(Q^{\prime}\right) \subseteq_{a} C\left(Q_{g}\right)$. We know that $C\left(Q_{g}\right) \subseteq_{a} \bigcup_{q \in \mathcal{B}(Q)} q$ from Proposition 3.2. Therefore $C\left(Q^{\prime}\right) \subseteq_{a} \bigcup_{q \in \mathcal{B}(Q)} q$. Since $Q^{\prime} \subseteq_{a} C\left(Q^{\prime}\right)$ and $C\left(Q^{\prime}\right) \subseteq_{a} \bigcup_{q \in \mathcal{B}(Q)} q$, we have $Q^{\prime} \subseteq_{a} \bigcup_{q \in \mathcal{B}(Q)} q$.

Let $x$ be a distinguished variable at the $i$ th position in the head of $C\left(Q^{\prime}\right)$ and suppose $x$ occurs at the $j$ th position of the $p$ th subgoal $S(\mathbf{u})$ of $C\left(Q^{\prime}\right)$. If $S(\mathbf{u})$ is among the subgoals in the minpart of $C\left(Q^{\prime}\right)$, then it must also be among the subgoals in the minpart of $C(Q)$. Hence the algorithm Generate-Query-Basis would have generated one or more queries whose combined effect is the query $C\left(Q_{g}\right)$, shown below,

$H(\mathbf{x})$ :- minpart, rest,$S\left(\mathbf{w}_{\mathbf{1}}, x, \mathbf{w}_{\mathbf{2}}\right)$.

(The variable $x$ occurs at the $j$ th position in the subgoal $S\left(\mathbf{w}_{\mathbf{1}}, x, \mathbf{w}_{\mathbf{2}}\right)$ and $\mathbf{w}_{\mathbf{1}}$ and $\mathbf{w}_{\mathbf{2}}$ are vectors of distinct variables that do not occur in $C(Q)$.) This corresponds to Step 2 of the algorithm where a new relation $S$ is added to the FROM clause and clauses of the form " $B$ TO $A$ " are added to the PROPAGATE clause to simulate the effect of $x$ propagating annotations to the output. We assume that $x$ occurs under the attribute $A$ in the output and $B$ is the attribute name of $x$ in $S$ in the named perspective. If $x$ occurs under another attribute $D$ in the output of $C\left(Q_{g}\right)$, there will be another query generated by Step 2 of the algorithm that propagates the annotations of $B$ to $D$. Hence, there is possible more than one $\mathrm{pSQL}$ query whose combined annotation propagation effect equals that of $C\left(Q_{g}\right)$.

It is easy to see that there is a homomorphism from $C\left(Q_{g}\right)$ to $C\left(Q^{\prime}\right)$ with the desired properties required by the fact shown above. The homomorphism is obtained by extending the homomorphism $h^{\prime}: C(Q) \rightarrow C\left(Q^{\prime}\right)$ which we know exists since $C(Q)=C\left(Q^{\prime}\right)$. The homomorphism $h^{\prime}$ is extended to $h^{\prime \prime}$ by mapping the $i$ th variable in $\mathbf{w}_{\mathbf{1}}$ to the corresponding $i$ th variable in $\mathbf{u}$ and the $i$ th variable in $\mathbf{w}_{\mathbf{2}}$ to the $(j+i)$ th variable in $\mathbf{u}$ (this is possible since $\mathbf{w}_{\mathbf{1}}$ and $\mathbf{w}_{\mathbf{2}}$ are distinct variables). Clearly, $h^{\prime \prime}$ satisfi es the conditions required by the above fact. If $S(\mathbf{u})$ are among the subgoals in rest 2 of $C\left(Q^{\prime}\right)$, we fi rst claim that a subgoal $S\left(\mathbf{u}^{\prime}\right)$, where the $j$ th variable of $u^{\prime}$ is $x$, must also occur among subgoals in the minpart of $Q^{\prime}$. With this, a similar argument presented before shows that there must be a homomorphism from a query $C\left(Q_{g}\right)$ to $C\left(Q^{\prime}\right)$ with the desired conditions required by the above fact and hence, $C\left(Q^{\prime}\right) \subseteq_{a} C\left(Q_{g}\right)$. Since the annotation propagation behavior of $C\left(Q_{g}\right)$ is equal to the combined annotation propagation effect of one or more queries in $\bigcup_{q \in \mathcal{B}(Q)} q$, we have $C\left(Q_{g}\right) \subseteq_{a} \bigcup_{q \in \mathcal{B}(Q)} q$.

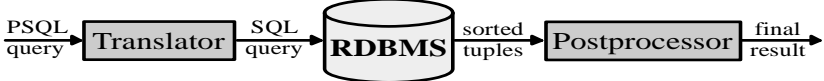

Figure 3: Architecture of our system.

We show next that if $S(\mathbf{u})$ are among the subgoals in rest 2 of $C\left(Q^{\prime}\right)$, there must exist such a subgoal $S\left(\mathbf{u}^{\prime}\right)$ among the minpart of $C\left(Q^{\prime}\right)$. Since there is a homomorphism $g$ from $C\left(Q^{\prime}\right)$ to the minimal query of $C\left(Q^{\prime}\right)$ and $g(x)=x$ (since $x$ is a distinguished variable), this implies that there must be a subgoal $S(\ldots x \ldots)$ among the subgoals in the minpart of $C\left(Q^{\prime}\right)$ such that $x$ occurs at the $j$ th position of this subgoal. We therefore conclude that $S\left(\mathbf{u}^{\prime}\right)$ exists.

Theorem 3.2 Let $Q$ be a $p S Q L$ query fragment with default-all propagation scheme. The algorithm GenerateQuery-Basis $(Q)$ returns a query basis of $Q$.

Proof. Let $\mathcal{E}(Q)$ denote the set of pSQL query fragments $q$ under the default propagation scheme such that the SQL query that corresponds to $q$ is equivalent to that of $Q$ (i.e., $S(q)=S(Q)$ ). Let $\mathcal{B}(Q)$ denote the result of running the algorithm Generate-Query-Basis on $Q$. By Lemma 3.1, $\bigcup_{q \in \mathcal{E}(Q)} q \subseteq \subseteq_{a} \bigcup_{q \in \mathcal{B}(Q)} q$. Since $\mathcal{B}(Q) \subseteq \mathcal{E}(Q)$, we immediately have $\bigcup_{q \in \mathcal{B}(Q)} q \subseteq a \quad \bigcup_{q \in \mathcal{E}(Q)} q$ and hence the result.

Proposition 3.3 Given a pSQL query fragment $Q$ with the default-all propagation scheme, the number of queries returned by Generate-Query-Basis $(Q)$ is polynomial in the size of $Q$. Furthermore, each query in Generate-Query$\operatorname{Basis}(Q)$ is polynomial in the size of $Q$.

An optmization Observe that the auxiliary pSQL queries overlap signifi cantly in the PROPAGATE clauses (e.g., see Figure 2); they differ only in the last (highlighted) propagation. In fact, we show that the non-highlighted propagations in the auxiliary queries are unnecessary (the details are omitted). Intuitively, they are unnecessary because these propagations are identical to the propagations of the representative query $Q_{0}$. Hence, in our optimized implementation of Generate-Query-Basis, these non-highlighted propagations are not generated in the auxiliary queries. We refer to our original implementation of algorithm GenerateQuery-Basis as the unoptimized implementation.

\section{System Architecture}

The architecture of our Annotation Management System is illustrated in Figure 3. We have two main modules: the translator module and the postprocessor module. The translator module takes as input a pSQL query and returns as output an SQL query (i.e., a union of SPJ queries) which is sent to the RDBMS. The SQL query is then executed by the RDBMS. The tuples that are returned by the RDBMS are sorted in a certain order and sent to the postprocessor module which merges annotations of identical cells of duplicate tuples together in one pass through the returned tuples. 


\subsection{A Naive Storage Scheme}

At present, we store our annotations using a naive storage scheme: we assume that every attribute $A$ of a relation scheme $R$ has an extra column $A_{a}$ that will be used to store annotations. We denote this new relation with extra columns as $R^{\prime}$. For example, a relation $R(A, B)$ will be represented as $R^{\prime}\left(A, A_{a}, B, B_{a}\right)$ in the naive storage scheme. Given a tuple $t$ in a relation of $R$, if $\left\{a_{1}, \ldots, a_{k}\right\}$ are the annotations associated with the location $(t, A)$, then there will be $k$ tuples $t_{1}, \ldots, t_{k}$ in $R^{\prime}$ such that $t_{i} . A_{a}=a_{i}$ for $i \in[1, k]$ and $t_{i} \cdot R=t, i \in[1, k]$ where $t_{i} \cdot R$ denotes the projection of $t_{i}$ on the attributes of $R$. For convenience, we sometimes use the relation name $R$ to refer to $R^{\prime}$. As an example, the two instances of $R$ shown below are both valid representations of the tuple (a $\left.\left\{a_{1}, a_{2}\right\}, \mathrm{b}\left\{b_{1}\right\}\right)$.

\begin{tabular}{|c|l|l|l|}
\hline$A$ & $A_{a}$ & $B$ & $B_{a}$ \\
\hline \hline $\mathrm{a}$ & $a_{1}$ & $\mathrm{~b}$ & $b_{1}$ \\
$\mathrm{a}$ & $a_{2}$ & $\mathrm{~b}$ & - \\
\hline
\end{tabular}

\begin{tabular}{|c|c|c|c|}
\hline$A$ & $A_{a}$ & $B$ & $B_{a}$ \\
\hline \hline $\mathrm{a}$ & $a_{1}$ & $\mathrm{~b}$ & - \\
$\mathrm{a}$ & $a_{2}$ & $\mathrm{~b}$ & - \\
$\mathrm{a}$ & $a_{2}$ & $\mathrm{~b}$ & $b_{1}$ \\
\hline
\end{tabular}

Observe that a query returns the same result regardless of the underlying storage instance used. In the case where every cell has a distinct annotation that denotes its address, then one could defi ne $R$ as a view defi nition of $R$ using the internal row identifi er used in many database systems such as Oracle and Postgres.

\subsection{The Translator}

The translator module takes as input a pSQL query $Q$ and translates $Q$ to an SQL query $Q^{\prime}$ against the naive storage scheme. A pSQL query with default or default-all propagation scheme is fi rst reformulated into one with a custom propagation scheme. A pSQL query with the custom propagation scheme is reformulated into an SQL query (i.e., a union of SPJ queries). The algorithm for reformulating a pSQL query fragment with default propagation scheme into a $\mathrm{pSQL}$ fragment with custom propagation scheme is described briefly at the end of Section 2.2. The algorithm for reformulating a pSQL query fragment with defaultall propagation scheme into a $\mathrm{pSQL}$ query fragment with custom propagation scheme is described by the GenerateQuery-Basis algorithm in Section 2.3. We describe next the algorithm for reformulating a pSQL query with custom propagation scheme into an SQL query.

Algorithm Custom-pSQL-To-SQL

Input: A pSQL query fragment $Q$ with custom propagation scheme.

Output: An SQL query $Q_{s}$ written against the naive schema.

Let $Q$ be a pSQL query fragment of the form shown in Definition 2.1 with a custom-propagatelist.

1. Generate intermediate $S Q L$ queries. Each intermediate SQL query retrieves annotations (as much as possible) from the naive schema according to the given query $Q$.

Let $Q_{0}$ be a query that is identical to $Q$ except that it does not have the PROPAGATE clause of $Q$.

For each output attribute $C$ of $Q$, create an empty bin for $C$. Denote this bin as $\operatorname{bin}(C)$. For each propagate clause " $s . B$
TO $C$ " in the custom-propagatelist of $Q$, add "s. $B{ }_{a}$ AS $C_{a}$ " to $\operatorname{bin}(C)$.

Let $\mathcal{Q}$ be the empty set of SQL queries. Repeat until all bins are empty:

Let $Q^{\prime}$ be a query that is identical to $Q_{0}$. For each output attribute $C$ of $Q$, if $\operatorname{bin}(C)$ is nonempty, remove a clause "s. $B_{a}$ AS $C_{a}$ " from $\operatorname{bin}(C)$ and add it to the selectlist of $Q^{\prime}$. If $\operatorname{bin}(C)$ is empty, we add 'NULL AS $C_{a}$ " to the selectlist of $Q^{\prime}$. Add $Q^{\prime}$ to $\mathcal{Q}$.

2. Generate a wrapper SQL query $Q_{s}$ for $\mathcal{Q}$. SELECT DISTINCT *

FROM

ORDER BY

$\left(Q_{1}\right.$ UNION $\cdots$ UNION $\left.Q_{n}\right)$ orderbylist

where $\mathcal{Q}=\left\{Q_{1}, \ldots, Q_{n}\right\}$ and orderbylist is the list of all output attributes in the selectlist of $Q$. The orderbylist is required so that the Postprocessor can merge annotations of identical tuples together with one pass over the result of $Q_{s}$.

3. Return $Q_{s}$.

Example 4.1 Consider the SWISS-PROT relation of Figure 1 and assume that there is an extra attribute Size. Suppose we have the following pSQL query $Q$ with custom propagation scheme written against SWISS-PROT:

SELECT $s$.ID AS ID, $s$. Desc AS Desc, $s$. Size AS Size,

FROM SWISS-PROT $s$

PROPAGATE $s$.ID TO Desc, $s$. Desc TO Desc, $s$.Size TO Size,

Observe that every tuple in SWISS-PROT will be emitted in such a way that the set of annotations associated with the Desc column of a tuple in the output is the union of annotations associated with both ID and Desc of the corresponding tuple in SWISS-PROT. Furthermore, the annotations associated with the Si ze column of a tuple are the same annotations associated with the $\mathrm{Size}$ column of the corresponding tuple in SWISS-PROT and the column ID of every tuple in the output does not carry any annotations.

In step 1 of algorithm Custom-pSQL-To-SQL, the following two intermediate $\mathrm{SQL}$ queries are generated since

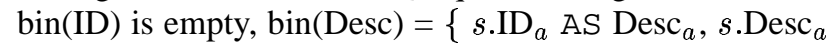
$\left.\mathrm{AS} \mathrm{Desc}_{a}\right\}$ and bin(Size $)=\left\{s \cdot \operatorname{Size}_{a} \mathrm{AS} \mathrm{Size}_{a}\right\}$.

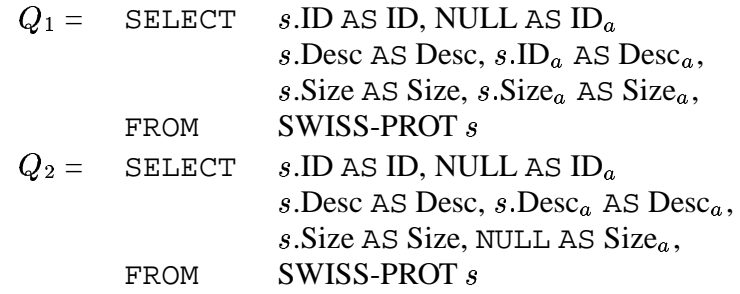

In step 2, the algorithm generates the following wrapper SQL query:

$$
\begin{array}{rll}
Q_{s}= & \text { SELECT DISTINCT } & * \\
& \text { FROM } & \left(Q_{1} \text { UNION } Q_{2}\right) \\
& \text { ORDER BY } & \text { ID, Desc, Size }
\end{array}
$$

Observe that $Q_{1}$ and $Q_{2}$ are unioned and the result is sorted according to the attributes in the selectlist of $Q$. The tuples are sorted according to the selectlist of $Q$ so that the Postprocessor can merge annotations associated with identical cells in the output of $Q$ in one pass over the result 

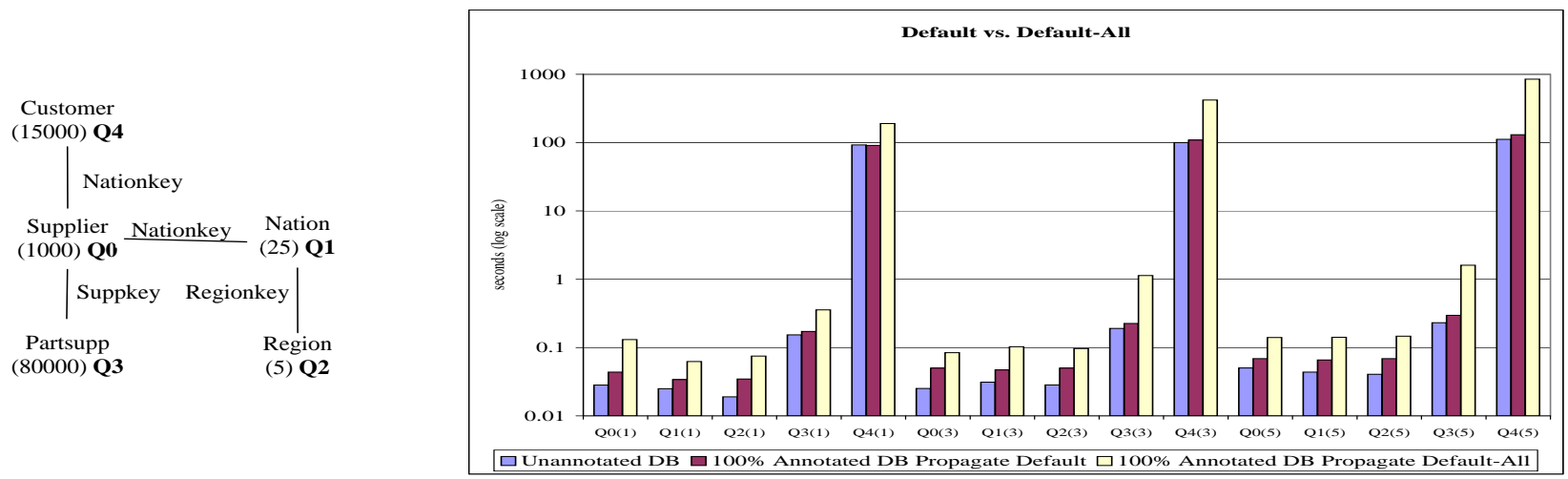

Figure 4: Queries used in our experiments and comparison in performance for $100 \mathrm{MB}, 100 \%$ annotated TPCH database.

of $Q_{s}$. Observe also that the number of SQL queries in $\mathcal{Q}$ is equal to the maximum bin size.

\subsection{The Postprocessor}

The Postprocessor scans the set of tuples returned by the RDBMS and unions together the annotations from duplicate tuples for proper display. This operation is done in linear time in the number and size of tuples retrieved, provided that the set of emitted tuples is already sorted. For example, if the postprocessor receives the fi rst table of Section 4.1 as input, it returns $\left\{\left(\mathrm{a}\left\{a_{1}, a_{2}\right\}, \mathrm{b}\left\{b_{1}\right\}\right)\right\}$.

\section{Experimental Evaluation}

We conducted several experiments to evaluate the feasibility of our annotation management system. Our main goal is to compare the performance of queries under different propagation schemes (default, default-all, or no propagation scheme (i.e., SQL queries)) and to compare the performance of queries when the number of annotations in a database is varied.

Setup We have implemented our system on top of Oracle 9i Enterprise Edition. For our experiments we used 100MB TPCH database (and subsequently 500MB and 1GB TPCH databases), which we call unannotated database. We have also modifi ed TPCH schema to conform to our naive storage scheme by adding an additional attribute for every attribute of every relation in the TPCH schema. We have created three different instances of the modifi ed TPCH database schema corresponding to $30 \%, 60 \%$ and $100 \%$ annotated databases. A $30 \%$ annotated database means $30 \%$ of the total number of cells in every relation instance of the database will contain one annotation. We ran queries of increasing join sizes to determine how well our system scales for this type of queries. (We did not use TPCH queries in our experiments because they include aggregates and nested queries.) The queries $Q_{0}, \ldots, Q_{4}$ denote queries with zero to four joins, respectively, and are shown on the left of Figure 4. For example, $Q_{2}$ denotes the query Supplier $\bowtie$ Nation $\bowtie$ Region with two joins, on the attributes Nationkey and Regionkey respectively. The cardinality of each relation is shown in brackets. Our experi- ments are conducted on a Pentium $4,2.8 \mathrm{GHz}$ machine with 1GB RAM.

Experiments We fi rst measure the performance of our system for queries under the default and default-all propagation scheme on the $100 \%$ annotated database. We have implemented and tested both optimized as well as unoptimized versions of our Generate-Query-Basis algorithm. For space reasons we present only our results obtained with the optimized version, as we observed that it consistently and signifi cantly outperforms the unoptimized version. We executed queries $Q_{i}(1), Q_{i}(3), Q_{i}(5), i \in[0,4]$, which denote queries with $i$ joins and one, three, and fi ve output attributes, respectively. We also executed the SQL query that corresponds to each of these queries on the unannotated database. The results are shown in Figure 4.

Figure 4 illustrates the execution time (the total time taken by the translator, RDBMS, and postprocessor to emit all tuples in the result) of each query for the default and default-all propagation schemes for the 100MB and 100\% annotated TPCH database. As expected, the execution time of each query under the default scheme (respectively, default-all scheme) increases slightly as more output attributes are emitted (see, for instance, $Q_{0}(1), Q_{0}(3)$, and $\left.Q_{0}(5)\right)$. The increase in time is due to longer execution time taken by Oracle as well as additional overhead incurred in postprocessing, as more attributes of different tuples need to be compared. Additionally, for the default-all scheme, the number of SPJ queries that are sent to Oracle increases $(2,4$, and 6 SPJ queries, respectively) as the number of output attributes increases. Table 1 provides the exact execution times of each query for $100 \%$ annotated database and the number of SPJ queries that are generated for the default all-scheme. We note that in the worst case, a query such as $Q_{4}(5)$ may run about 8 times slower than both the query with default scheme and the actual SQL query. This is not unexpected, however, as there are $6 \mathrm{SPJ}$ queries, each with four joins, that are generated and sent to Oracle for $Q_{4}(5)$, instead of 1 . In the best case (see $Q_{4}(1)$ ), a query with default-all scheme runs about twice as slow than the same query with default scheme. We note however that for the default scheme, the execution times of pSQL queries are comparable to those of SQL queries. On 


\begin{tabular}{|c|c|c|c|c|c|c|c|c|c|}
\hline Query & Unannotated & $30 \%$ Def & $30 \%$ Def-All & $60 \%$ Def & $60 \%$ Def-All & $100 \%$ Def & $100 \%$ Def-All & \#pSQL & \#SPJ \\
\hline$Q_{0}(1)$ & 0.0282 & 0.0374 & 0.1316 & 0.0408 & 0.125 & 0.0438 & 0.1308 & 2 & 2 \\
\hline$Q_{1}(1)$ & 0.025 & 0.0344 & 0.0658 & 0.034 & 0.072 & 0.034 & 0.0624 & 2 & 2 \\
\hline$Q_{2}(1)$ & 0.019 & 0.0312 & 0.0722 & 0.0342 & 0.0748 & 0.0346 & 0.075 & 2 & 2 \\
\hline$Q_{3}(1)$ & 0.1532 & 0.1752 & 0.3622 & 0.1688 & 0.3594 & 0.1718 & 0.356 & 2 & 2 \\
\hline$Q_{4}(1)$ & 92.4604 & 92.2198 & 190.7312 & 91.7214 & 190.826 & 91.2248 & 190.3552 & 2 & 2 \\
\hline$Q_{0}(3)$ & 0.0252 & 0.0468 & 0.0848 & 0.0468 & 0.084 & 0.05 & 0.084 & 4 & 4 \\
\hline$Q_{1}(3)$ & 0.0312 & 0.0502 & 0.0968 & 0.0374 & 0.0968 & 0.047 & 0.103 & 4 & 4 \\
\hline$Q_{2}(3)$ & 0.0284 & 0.0502 & 0.1002 & 0.0562 & 0.0998 & 0.05 & 0.0968 & 4 & 4 \\
\hline$Q_{3}(3)$ & 0.191 & 0.219 & 1.1186 & 0.2216 & 1.1188 & 0.225 & 1.1314 & 4 & 4 \\
\hline$Q_{4}(3)$ & 100.0106 & 113.4292 & 422.6232 & 108.2372 & 424.6066 & 109.012 & 419.5722 & 4 & 4 \\
\hline$Q_{0}(5)$ & 0.0502 & 0.069 & 0.1372 & 0.072 & 0.1438 & 0.069 & 0.1404 & 6 & 6 \\
\hline$Q_{1}(5)$ & 0.0438 & 0.0654 & 0.138 & 0.0718 & 0.1312 & 0.0658 & 0.1412 & 6 & 6 \\
\hline$Q_{2}(5)$ & 0.0406 & 0.0662 & 0.1498 & 0.0658 & 0.1468 & 0.0688 & 0.1466 & 6 & 6 \\
\hline$Q_{3}(5)$ & 0.231 & 0.287 & 1.6128 & 0.2908 & 1.6096 & 0.2968 & 1.6064 & 6 & 6 \\
\hline$Q_{4}(5)$ & 111.8918 & 131.3138 & 858.8238 & 130.5282 & 836.5362 & 130.6594 & 850.6284 & 6 & 6 \\
\hline
\end{tabular}

Table 1: The execution times of each query for each database and propagation scheme. The columns "\#pSQL" and "\#SPJ" denote the size of the query basis and number of SPJ queries that are generated, respectively, for the default-all scheme.

the average, the pSQL queries with default scheme that we experimented with took around $40 \%$ more time to execute than their corresponding SQL queries, and at best the execution time of a pSQL query with default scheme is the same as the execution time of its corresponding SQL query (e.g., $\left.Q_{4}(1)\right)$. For larger databases (500MB and 1GB), the pSQL queries with default scheme took only about $18 \%$ more time to execute than their corresponding SQL queries on the average (these results are not shown).

Subsequently, we also conducted the same experiments on $30 \%$ and $60 \% 100 \mathrm{MB}$ annotated databases. The results are tabulated in Table 1 . We observe that the execution time of each query increases only slightly across different databases. For example, the execution time of each query for both default and default-all scheme increases marginally when the number of annotations in the database is doubled from $30 \%$ annotations to $60 \%$. We also remark that for the default-all scheme there is no increase in the number of pSQL and SPJ queries that are generated when the number of joins increases because the attributes that are selected do not participate in the joins. The number of pSQL and SPJ queries that are generated increases when the number of output attributes increases and they increase linearly. The execution times of $Q_{1}(j), j \in[1,3,5]$, decreases slightly when compared with $Q_{0}(j)$ because a join on a small relation has been made.

We also ran the same set of experiments (results are not shown) on $500 \mathrm{Mb}$ and $1 \mathrm{~GB}$ TPCH databases with $30 \%$, $60 \%$ and $100 \%$ annotations and we observed the same trend as in Figure 4. All our results indicate that the time required to translate the queries is insignifi cant when compared to the execution time of the queries and the postprocessing time of the queries is proportional to the number and size of emitted tuples. Also, the execution times of default queries are comparable to the performance of SQL queries since only one SPJ is generated.

\section{Discussion}

So far, our pSQL queries do not allow aggregates and bag semantics (i.e., the DISTINCT keyword must be present).
We discuss briefly next how we might extend pSQL to handle aggregates and bag queries as well.

Aggregates For the default propagation scheme, if a pSQL query contains aggregates such as count, sum, and average, we assume the semantics that no annotations are associated with the result of these aggregates, since these aggregate values are not copied from any source values. However, for aggregates such as $\min (a)$ and $\max (a)$, where $a$ is an attribute name, our semantics is that the annotations associated with the location of the resulting min (or max) value are the union of all annotations of the corresponding $a$-values whose value equals to the $\min$ (or max) value. It remains to investigate whether the default-all propagation scheme for $\mathrm{pSQL}$ queries with aggregates can be achieved. Bag semantics It is known from [8] that two conjunctive queries are equivalent under bag semantics if and only if they are isomorphic. This result of [8] implies that to propagate annotations for a pSQL query under the default-all propagation scheme and bag semantics, it suffi ces to generate only the representative query of that $\mathrm{pSQL}$ query in Algorithm Generate-Query-Basis. To handle bag queries, however, the naive storage scheme can no longer be used since the multiplicity of a tuple in this storage scheme depends on the number of annotations that are associated with that tuple. An alternative storage scheme that does not modify the original relation is needed (e.g., store every annotation and its location in a separate relation). To propagate annotations under the default-all propagation scheme and bag semantics for unions of conjunctive queries, however, it remains to fi rst provide a characterization of bag equivalence for unions of conjunctive queries.

\section{Conclusion and Future Work}

We have described an implementation of an annotation management system where different propagation schemes can be used. Insofar, our system only supports annotations on attributes of tuples. We would like to extend our system to handle annotations on tuples or relations and, in general, to handle annotations on hierarchical data, such as XML. In our current system, annotations are propagated based on where-provenance. In addition, we would like 
to extend our system to propagate annotations based on why-provenance, which will provide reasons to why a tuple is in the output. The default-all propagation scheme returns the union of all annotations of an output location returned by all equivalent queries. Conceivably, there could be a complementary propagation scheme that returns the set of all annotations in an output location if it occurs in the same output location in the results of all equivalent queries. It remains to be investigated whether a query basis can be generated for such propagation scheme. The performance of our annotation management system on other storage schemes also needs to be investigated. It would also be interesting to investigate opportunities for optimizations on the generated SQL queries.

Acknowledgements We thank Xinyu Hua for her help during the initial implementation of this system and Ariel Fuxman for helpful suggestions.

\section{References}

[1] S. Abiteboul, R. Hull, and V. Vianu. Foundations of Databases. Addison Wesley Publishing Co, 1995.

[2] R. Apweiler, A. Bairoch, C. Wu, W. Barker, B. Boeckmann, S. Ferro, E. Gasteiger, H. Huang, R. Lopez, M. Magrane, M. Martin, D. Natale, C. O'Donovan, N. Redaschi, and L. Yeh. Uniprot: the universal protein knowledgebase. $\mathrm{Nu}$ cleic Acids Research, 32:D115-D119, 2004.

[3] A. Bairoch and R. Apweiler. The SWISS-PROT protein sequence database and its supplement TrEMBL. Nucleic Acids Research, 28:45-48, 2000.

[4] P. Bernstein and T. Bergstraesser. Meta-Data Support for Data Transformations Using Microsoft Repository. IEEE Data Engineering Bulletin, 22(1):9-14, 1999.

[5] biodas.org. http://biodas.org.

[6] P. Buneman, S. Khanna, and W. Tan. Why and Where: A Characterization of Data Provenance. In Proceedings of the International Conference on Database Theory (ICDT), pages 316-330, London, United Kingdom, 2001.

[7] P. Buneman, S. Khanna, and W. Tan. On Propagation of Deletions and Annotations Through Views. In Proceedings of the ACM Symposium on Principles of Database Systems (PODS), pages 150-158, Wisconsin, Madison, 2002.

[8] S. Chaudhuri and M. Y. Vardi. Optimization of real conjunctive queries. In Proceedings of the ACM Symposium on Principles of Database Systems (PODS), pages 59-70, Washington, DC, 1993.

[9] Y. Cui, J. Widom, and J. Wiener. Tracing the Lineage of View Data in a Warehousing Environment. ACM Transactions on Database Systems (TODS), 25(2):179-227, 2000.

[10] DBCAT, The Public Catalog of Databases. http://www.infobiogen.fr/services/dbcat/, cited 5 June 2000.

[11] D. E. Denning, T. F. Lunt, R. R. Schell, W. R. Shockley, and M. Heckman. The SeaView Security Model. In IEEE Symposium on Security and Privacy, pages 218-233, Washington, DC, 1988.
[12] R. Dowell. A Distributed Annotation System. Technical report, Department of Computer Science, Washington University in St. Louis, 2001.

[13] S. Jajodia and R. S. Sandhu. Polyinstantiation integrity in multilevel relations. In IEEE Symposium on Security and Privacy, pages 104-115, Oakland, California, 1990.

[14] J. Kahan, M. Koivunen, E. Prud'Hommeaux, and R. Swick. Annotea: An open rdf infrastructure for shared web annotations. In Proceedings of the International World Wide Web Conference $(W W W 10)$, pages 623-632, Hong Kong, China, 2001.

[15] A. Kementseitsidis, M. Arenas, and R. J. Miller. Mapping Data in Peer-to-Peer Systems: Semantics and Algorithmic Issues. In Proceedings of the ACM SIGMOD International Conference on Management of Data (SIGMOD), pages 325-336, San Diego, CA, 2003.

[16] W. J. Kent, C. W. Sugnet, T. S. Furey, K. M. Roskin, T. H. Pringle, A. M. Zahler, and D. Haussler. The Human Genome Browser at UCSC. Genome Research, 12(5):996-1006, 2002.

[17] D. LaLiberte and A. Braverman. A Protocol for Scalable Group and Public Annotations. In Proceedings of the International World Wide Web Conference(WWW3), Darmstadt, Germany, 1995.

[18] T. Lee, S. Bressan, and S. Madnick. Source Attribution for Querying Against Semi-structured Documents. In Workshop on Web Information and Data Management (WIDM), Washington, DC, 1998.

[19] A. C. Myers and B. Liskov. A decentralized model for information control. In Proceedings of the ACM Symposium on Operating Systems Principles (SOSP), pages 129-142, Saint-Malo, France, 1997.

[20] T. A. Phelps and R. Wilensky. Multivalent Annotations. In Proceedings of the First European Conference on Research and Advanced Technology for Digital Libraries, pages 287303, Pisa, Italy, 1997.

[21] T. A. Phelps and R. Wilensky. Multivalent documents. Proceedings of the Communications of the Association for Computing Machinery (CACM), 43(6):82-90, 2000.

[22] T. A. Phelps and R. Wilensky. Robust intra-document locations. In Proceedings of the International World Wide Web Conference( $W W W 9$ ), pages 105-118, Amsterdam, Netherlands, 2000.

[23] M. A. Schickler, M. S. Mazer, and C. Brooks. Pan-Browser Support for Annotations and Other Meta-Information on the World Wide Web. In Proceedings of the International World Wide Web Conference(WWW5), Paris, France, 1996.

[24] W. Tan. Containment of relational queries with annotation propagation. In Proceedings of the International Workshop on Database and Programming Languages (DBPL), Potsdam, Germany, 2003.

[25] W3C. Annotea Project. http://www.w3.org/2001/Annotea.

[26] Y. R. Wang and S. E. Madnick. A Polygen Model for Heterogeneous Database Systems: The Source Tagging Perspective. In Proceedings of the International Conference on Very Large Data Bases (VLDB), pages 519-538, Brisbane, Queensland, Australia, 1990. 